\title{
On Local Fairing Algorithm for Cubic B-spline with the Second Discrete Curvature
}

\author{
Cheng Cheng ${ }^{1,2, a}$, Yu Desheng ${ }^{\text {1,b }}$ \\ ${ }^{1}$ School of Mathematics and Information Science, Nanchang Hangkong University, Nanchang \\ 330063, China \\ ${ }^{2}$ Mathematical Section, Huangmei Number 1 High School, Huangmei County, Hubei Province \\ 330063, China \\ ayixunxiangfan@163.com, ${ }^{b}$ yuds17 @ 163.com
}

\begin{abstract}
Keywords: discrete curvature, differential, fairing, cubic B-spline.
Abstract. In this paper, a new discrete curvature-the second discrete curvature is introduced. It is obtained that the curvatures of a cubic B-spline curve at its joining points are "proportional to" the second discrete curvatures of its corresponding control points about the node vector, and the second discrete curvature has similar properties but be more accurate than discrete curvature. The fairing algorithm for cubic B-spline based on the second discrete curvature is given. With this algorithm curves are faired through adjusting the second discrete curvature of the corresponding control points directly, thus the fairing process is more concise and has stronger geometrical intuition. It is showed that the algorithm can get better fairing effects through experiment examples.
\end{abstract}

\section{Introduction}

Fairing of curves and surfaces is an important topic of Computer Aided Design investigation. It has important theoretical and practical value. A lot of researches have been made and some effective fairing algorithms are obtained in this field. The algorithms can mainly be divided into three categories: Curvature-based fairing algorithms, Energy-based fairing algorithms and Curvature and energy-combined fairing algorithms. Curvature-based fairing algorithms, such as Poliakoff's automatic fairing algorithm [1], MCV fairing algorithm [2], Li's target curvature algorithm [3] and Glen's curvature fairing method [4], mainly adjust the spline control points of curves and surfaces (data points) to ensure that their appropriate curvature maps are continuous and monotonous; Energy-based fairing algorithms, such as least square fairing method [5], energy fairing method [6], and Robet's shape preserving interpolation fairing algorithm [7], generally based on physical deformation energy function, find the smallest physical deformation energy curves and surfaces under some geometric and non-geometric constraints; Curvature and energy-combined faring algorithms, such as local energy fairing algorithm [8], basis spline fairing algorithm [9], Zhang's minimum energy fairing algorithm [10], determine the areas to be faired mainly through curvature at first, and then fair them with energy minimizing methods. There are some special fairing algorithms, but to our knowledge, researches on discrete curvature-based fairing algorithms [11] through adjusting the geometric positions of control points directly are still rare.

Since B-spline curves have the properties that they can be described mathematically with control points, their shapes can be reflected by their control polygons, and their shape modification can be achieved by adjusting control points, so if we can fair the curves and surfaces through modifying control points of B-spline, their faring process will be more direct. To this aims, based on the studies of relation between the curvatures at the nodes of B- spline and discrete curvatures at their corresponding control points, this paper introduce a new discrete curvature - the second discrete curvature. It is obtained that the curve curvatures at nodes of B-spline curves and the second discrete curvatures at the corresponding control points are proportional, and the fairing algorithm based on the second discrete curvature for B-spline curve is given. Thus through adjusting the second discrete curvatures at control points properly, the fairing of B-spline curve segments at the endpoints and the purposes of fairing the 
B-spline curves can be achieved. So the fairing processes are more concise and have more geometric intuitions, and the results of [12] are generated.

\section{Concept and property of the second discrete curvature}

\section{Concept of discrete curvature}

Give a sequence of points planar $\boldsymbol{Q}_{i}\left(x_{i}, y_{i}\right)(i=0,1, \mathrm{~L}, n)$ which don't coincide. Let $L_{i}=\left\|\boldsymbol{Q}_{i}-\boldsymbol{Q}_{i-1}\right\|$ be the distance between $\boldsymbol{Q}_{i-1}$ and $\boldsymbol{Q}_{i}$, then the discrete curvature at point $\boldsymbol{Q}_{i}$ is defined as [13]

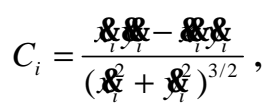

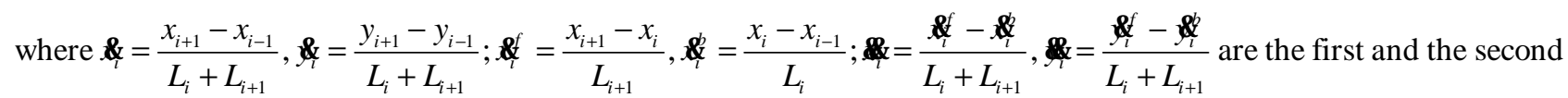
derivatives of coordinates to the line segment distances at point $\boldsymbol{Q}_{i}$.

Theorem 1 Let $\boldsymbol{Q}_{i}\left(x_{i}, y_{i}\right)(i=0,1, \mathrm{~L}, n)$ be a sequence of planar points which don't coincide, $M_{i}=\left\|\boldsymbol{Q}_{i+1}-\boldsymbol{Q}_{i-1}\right\|$ be the distance between $\boldsymbol{Q}_{i-1}$ and $\boldsymbol{Q}_{i+1}, D_{\boldsymbol{Q}_{i-1} Q_{i} Q_{i+1}}$ be the directed area of triangle $\boldsymbol{Q}_{i-1} \boldsymbol{Q}_{i} \boldsymbol{Q}_{i+1}[14]$, then the discrete curvature at point $\boldsymbol{Q}_{i}$ is

$$
C_{i}=\frac{2\left(L_{i}+L_{i+1}\right)^{2} D_{Q_{i-1} Q_{i+1}}}{L_{i} L_{i+1} M_{i}^{3}} .
$$

Proof Formula (2) is obtained by substituting the first and the second derivatives of coordinates to the line segment distances at point $\boldsymbol{Q}_{i}$ into formula (1) and then simplifying.

Remark 1 For the two end points $\boldsymbol{Q}_{0}, \boldsymbol{Q}_{n}$, only add two vertices $\boldsymbol{Q}_{-1}=2 \boldsymbol{Q}_{0}-\boldsymbol{Q}_{1}$ and $\boldsymbol{Q}_{n+1}=2 \boldsymbol{Q}_{n}-\boldsymbol{Q}_{n-1}$, and substituting $D_{Q_{-1} Q_{0} Q_{1}}$ and $D_{Q_{n-1} Q_{n} Q_{n+1}}$ as the corresponding directed areas of the triangles, then the discrete curvatures of the two boundary points $\boldsymbol{Q}_{1}, \boldsymbol{Q}_{n}$ can also be calculated by formula (2) respectively.

\section{Basic concept of the second discrete curvature}

Let $\Gamma^{(0)}=\boldsymbol{Q}_{0} \boldsymbol{Q}_{1} \mathrm{~L} \boldsymbol{Q}_{n}$ be a planar polygon, and denote $\boldsymbol{Q}_{j}^{(0)}=\boldsymbol{Q}_{j}(j=0,1, \mathrm{~L}, n)$. Cutting the corner by dividing $\boldsymbol{Q}_{j-1}^{(0)} \boldsymbol{Q}_{j}^{(0)}$ with proportion $\mu_{i-1}, \lambda_{i}\left(\mu_{i-1}, \lambda_{i}>0, \mu_{i-1}+\lambda_{i}<1\right)$, then two new vertices at left and right sides of $\boldsymbol{Q}_{j}^{(0)}$ are obtained [15]

$$
\left\{\begin{array}{l}
\boldsymbol{Q}_{2 j-1}^{(1)}=\left(1-\lambda_{j}\right) \boldsymbol{Q}_{j}^{(0)}+\lambda_{j} \boldsymbol{Q}_{j-1}^{(0)}, j=0,1, \mathrm{~L}, n, \\
\boldsymbol{Q}_{2 j}^{(1)}=\mu_{j} \boldsymbol{Q}_{j+1}^{(0)}+\left(1-\mu_{j}\right) \boldsymbol{Q}_{j}^{(0)}
\end{array}\right.
$$

where $\boldsymbol{Q}_{n+1}^{(0)}=\boldsymbol{Q}_{0}^{(0)}, \boldsymbol{Q}_{-1}^{(0)}=\boldsymbol{Q}_{n}^{(0)}$ if $\Gamma^{(0)}$ is closed, and $\boldsymbol{Q}_{-1}^{(0)}=\boldsymbol{Q}_{0}^{(0)}, \boldsymbol{Q}_{n+1}^{(0)}=\boldsymbol{Q}_{n}^{(0)}$ if $\Gamma^{(0)}$ is open. Connecting these new vertices $\boldsymbol{Q}_{-1}^{(1)}, \boldsymbol{Q}_{0}^{(1)}, \mathrm{L}, \boldsymbol{Q}_{2 n}^{(1)}$ successively, we obtain a polygon $\Gamma^{(1)}=\boldsymbol{Q}_{-1}^{(1)} \boldsymbol{Q}_{0}^{(1)} \mathrm{L} \boldsymbol{Q}_{2 n}^{(1)}$, then $\Gamma^{(1)}$ is called the generating polygon from cutting $\Gamma^{(0)}$ 's corners.

Definition 1 Let $\Gamma^{(0)}=\boldsymbol{Q}_{0} \boldsymbol{Q}_{1} \mathrm{~L} \boldsymbol{Q}_{n}$ be the control polygon of a cubic B-spline curve, cutting $\Gamma^{(0)}$ 's corners with proportions $\lambda_{i}=\frac{u_{i+3}-u_{i+2}}{u_{i+3}-u_{i}}, \mu_{i}=\frac{u_{i+2}-u_{i+1}}{u_{i+4}-u_{i+1}}$, then

$$
K_{i}=D_{\boldsymbol{Q}_{i-1} Q_{i} \boldsymbol{Q}_{i+1}} /\left\|\lambda_{i}\left(\boldsymbol{Q}_{i}-\boldsymbol{Q}_{i-1}\right)+\mu_{i}\left(\boldsymbol{Q}_{i+1}-\boldsymbol{Q}_{i}\right)\right\|^{3}
$$

is called the second curvature of the cubic B-spline at vertices $\boldsymbol{Q}_{i}$.

As a special case, when $\Gamma^{(0)}$ is the control polygon of a cubic uniform B-spline curve, i.e. the distances of all node intervals $\Delta u_{i}=u_{i+1}-u_{i}$ are equal to a constant, then the second curvature of the cubic B-spline at vertices $\boldsymbol{Q}_{i}$ is

$$
K_{i}=27 D_{Q_{i-1} Q_{i} Q_{i+1}} / M_{i}^{3} .
$$

Definition 2 The first and the second differences at vertices $\boldsymbol{Q}_{i}$ of a cubic B-spline curve are defined respectively as

$$
\Delta K_{0}=0, \Delta K_{i}=K_{i}-K_{i-1}, i=1, \mathrm{~K}, n
$$


where $\tau_{i}=L_{i+1} /\left(L_{i}+L_{i+1}\right)$.

$$
D_{0}=D_{n}=0, D_{i}=\tau_{i} K_{i-1}+\left(1-\tau_{i}\right) K_{i+1}-K_{i}, i=1, \mathrm{~L}, n-1,
$$

\section{Basic properties of the second discrete curvature}

Let $\boldsymbol{Q}_{i-1}^{\circ}=\lambda_{i} \boldsymbol{Q}_{i-1}+\left(1-\lambda_{i}\right) \boldsymbol{Q}_{i}, \boldsymbol{Q}_{i}^{\circ}=\left(1-\mu_{i}\right) \boldsymbol{Q}_{i}+\mu_{i} \boldsymbol{Q}_{i+1}, \sigma_{i}=L_{i+1} / L_{i}, l_{i}=\left\|\boldsymbol{Q}_{i}-\boldsymbol{Q}_{i-1}^{\circ}\right\|, l_{i+1}=\left\|\boldsymbol{Q}_{i}-\boldsymbol{Q}_{i}^{\circ}\right\|$ be the distances between $\boldsymbol{Q}_{i}$ and $\boldsymbol{Q}_{i}^{\circ}, \boldsymbol{Q}_{i}$ and $\boldsymbol{Q}_{i-1}^{\circ}$ respectively, $\alpha_{i}=<l_{i}, l_{i+1}>$ be the angle between lines $l_{i}$ and $l_{i+1}$. Then we have the following:

Proposition 1 The relation between the discrete curvature and the second discrete curvature of a sequence of points $\boldsymbol{Q}_{i}(i=0,1, \mathrm{~L}, n)$ on plane $R^{2}$ which don't coincide is

$$
C_{i}=2 K_{i}\left(L_{i}+L_{i+1}\right)^{2}\left\|\mathscr{Q}_{i-1}^{\circ}-Q_{i}^{\prime}\right\|^{3} / L_{i} L_{i+1} M_{i}^{3} .
$$

Proposition 2 The second curvatures at two overlapping control points and the curvatures at the corresponding point on B-spline curve are zero, but the discrete curvatures at those points do not exist.

In fact, if two control points coincide, namely $\boldsymbol{Q}_{i}=\boldsymbol{Q}_{i+1}$, then the three points $\boldsymbol{Q}_{i-1}, \boldsymbol{Q}_{i}, \boldsymbol{Q}_{i+1}$ are collinear and $\mathscr{Q}_{i}^{\circ}=Q_{i}$, thus

$$
D_{\boldsymbol{Q}_{i-1} \boldsymbol{Q}_{i} \boldsymbol{Q}_{i+1}}=0,\left\|\boldsymbol{Q}_{i}^{\circ}-\boldsymbol{Q}_{i-1}^{\circ}\right\|=\lambda_{i}\left\|\boldsymbol{Q}_{i}-\boldsymbol{Q}_{i-1}\right\| .
$$

Therefore, the establishment of the above is concluded.

Proposition 3 Let $L_{i}=L_{i}^{\prime}, L_{i+1}=L_{i+1}^{\prime}, \alpha_{i}<\alpha_{i}^{\prime}$, then the relation of the two second discrete curvatures at control point $\boldsymbol{Q}_{i}$ is $K_{i}>K_{i}^{\prime}$.

In fact,

$$
K_{i}=\frac{D_{Q_{i-1} Q_{Q} Q_{i+1}}}{\left\|\mathscr{Q}_{i-1}^{\circ}-\boldsymbol{Q}_{i}^{\prime}\right\|^{3}}=\frac{2 L_{i} L_{i+1} \sin \alpha_{i}}{\left(l_{i}^{2}+l_{i+1}^{2}-2 l_{i} l_{i+1} \cos \alpha_{i}\right)^{3 / 2}}>\frac{2 L_{i} L_{i+1} \sin \alpha_{i}^{\prime}}{\left(l_{i}^{2}+l_{i+1}^{2}-2 l_{i} l_{i+1} \cos \alpha_{i}^{\prime}\right)^{3 / 2}}=K_{i}^{\prime} .
$$

Proposition 4 Let $L_{i}<L_{i}^{\prime}, L_{i+1}<L_{i+1}^{\prime}, \alpha_{i}=\alpha_{i}^{\prime}$ and $L_{i+1} / L_{i}=L_{i+1}^{\prime} / L_{i}^{\prime}$, then the relation of the two second discrete curvatures at control point $\boldsymbol{Q}_{i}$ is $K_{i}>K_{i}^{\prime}$.

In fact, in this case we have $l_{i}=\lambda_{i} L_{i}, l_{i+1}=\mu_{i} L_{i+1}$, thus

$$
\frac{M_{i}}{\left\|Q_{i-1}^{\text {o }}-\boldsymbol{Q}_{i}\right\|}=\frac{\sqrt{L_{i}^{2}+L_{i+1}^{2}-2 L_{i} L_{i+1} \cos \alpha_{i}}}{\sqrt{l_{i}^{2}+l_{i+1}^{2}-2 l_{i} l_{i+1} \cos \alpha_{i}}}=\sqrt{\frac{1+\sigma_{i}^{2}-2 \sigma_{i} \cos \alpha_{i}}{\lambda_{i}^{2}+\mu_{i}^{2} \sigma_{i}^{2}-2 \lambda_{i} \mu_{i} \sigma_{i} \cos \alpha_{i}}} .
$$

Because $\Delta \boldsymbol{Q}_{i-1} \boldsymbol{Q}_{i} \boldsymbol{Q}_{i+1} \quad \Delta \boldsymbol{Q}_{i-1}^{\prime} \boldsymbol{Q}_{i} \boldsymbol{Q}_{i+1}^{\prime}$, we have $\sigma_{i}=\sigma_{i}^{\prime}, \lambda_{i}=\lambda_{i}^{\prime}, \mu_{i}=\mu_{i}^{\prime}$. Thus

So

$$
\frac{M_{i}^{3}}{\left\|\mathscr{Q}_{i-1}^{\prime o}-\mathcal{Q}_{i}^{\prime}\right\|^{3}}=\frac{M_{i}^{\prime 3}}{\left\|\mathscr{Q}_{i-1}^{6}-\mathcal{Q}_{i}^{\prime}\right\|^{3}} \text {. }
$$

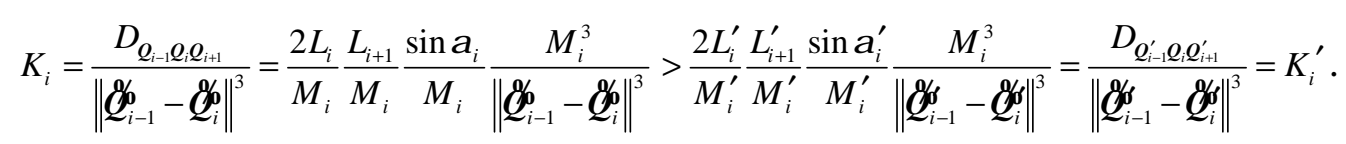

Proposition 5 The relation of the first and the second differences of the second discrete curvatures at control point $Q_{\mathrm{i}}$ is

$$
D_{i}=\frac{L_{i} L_{i+1}}{L_{i}+L_{i+1}}\left(\frac{\Delta K_{i+1}}{L_{i+1}}-\frac{\Delta K_{i}}{L_{i}}\right) .
$$

From this, it is conclude that $D_{i}=0$ shows that the ratio of the first difference of the first discrete curvature and the length of the chord varies uniformly; the smaller $\left|D_{i}\right|$, more evenly the changes of the ratio of the first difference between the first discrete curvature and the length of the chord. 


\title{
Smoothing principle based on the second discrete curvatures
}

\section{Relation between the curvatures at the endpoints of cubic B-spline curve segments and the second discrete curvatures of their control points}

\author{
Let $p(u)=\sum_{i=0}^{n} \boldsymbol{Q}_{i} N_{i+3}, u \in\left[u_{3}, u_{n+1}\right]$ be a cubic B-spline curve determined by $n+1$ control points
}

$\boldsymbol{Q}_{0}, \boldsymbol{Q}_{1}, \mathrm{~L}, \boldsymbol{Q}_{n}$. When $u \in\left[u_{i+2}, u_{i+3}\right](i=1,2, \mathrm{~L}, n-2)$, the curve segment is determined by four control points $\boldsymbol{Q}_{i-1}, \boldsymbol{Q}_{i}, \boldsymbol{Q}_{i+1}, \boldsymbol{Q}_{i+2}$, and denoted by $\boldsymbol{p}_{i}(u)(i=1,2, \mathrm{~L}, n-2)$. We have the following

Theorem 2 Let $\kappa_{i}$ be the curvature at the endpoint $\boldsymbol{p}\left(u_{i+2}\right)$ of a cubic B-spline curve segment $\boldsymbol{p}_{i}(u)(i=1,2, \mathrm{~L}, n-2), K_{i}$ be the second discrete curvature at its corresponding control vertices $\boldsymbol{Q}_{i}$, then

$$
K_{i}=\frac{3\left(u_{i+3}-u_{i}\right)\left(u_{i+4}-u_{i+1}\right)}{4\left(u_{i+3}-u_{i+1}\right)^{2}} \kappa_{i} .
$$

Proof The curvature at the endpoint $\boldsymbol{p}\left(u_{i+2}\right)$ of the cubic B-spline curve segment is

$$
\kappa_{i}=\frac{\boldsymbol{p}^{\prime}\left(u_{i+2}\right) \times \boldsymbol{p}^{\prime \prime}\left(u_{i+2}\right)}{\left|\boldsymbol{p}^{\prime}\left(u_{i+2}\right)\right|^{3}} .
$$

By the properties of cubic non-uniform B-spline curves, we know the tangent vector and the second derivative vector at the point $\boldsymbol{p}\left(u_{i+2}\right)$ of $\boldsymbol{p}(u)$ are

Thus

$$
\begin{aligned}
& \boldsymbol{p}^{\prime}\left(u_{i+2}\right)=\frac{3\left(u_{i+3}-u_{i+2}\right)}{\left(u_{i+3}-u_{i}\right)\left(u_{i+3}-u_{i+1}\right)}\left(\boldsymbol{Q}_{i}-\boldsymbol{Q}_{i-1}\right)+\frac{3\left(u_{i+2}-u_{i+1}\right)}{\left(u_{i+4}-u_{i+1}\right)\left(u_{i+3}-u_{i+1}\right)}\left(\boldsymbol{Q}_{i+1}-\boldsymbol{Q}_{i}\right), \\
& \boldsymbol{p}^{\prime \prime}\left(u_{i+2}\right)=\frac{-6}{\left(u_{i+3}-u_{i}\right)\left(u_{i+3}-u_{i+1}\right)}\left(\boldsymbol{Q}_{i}-\boldsymbol{Q}_{i-1}\right)+\frac{6}{\left(u_{i+4}-u_{i+1}\right)\left(u_{i+3}-u_{i+1}\right)}\left(\boldsymbol{Q}_{i+1}-\boldsymbol{Q}_{i}\right) .
\end{aligned}
$$

$$
\left|\boldsymbol{p}^{\prime}\left(u_{i+2}\right)\right|=\frac{3}{u_{i+3}-u_{i+1}} \mid \frac{u_{i+3}-u_{i+2}}{u_{i+3}-u_{i}}\left(\boldsymbol{Q}_{i}-\boldsymbol{Q}_{i-1}\right)+\frac{u_{i+2}-u_{i+1}}{u_{i+4}-u_{i+1}}\left(\boldsymbol{Q}_{i+1}-\boldsymbol{Q}_{i}\right) \|, \boldsymbol{p}^{\prime}\left(u_{i+2}\right) \times \boldsymbol{p}^{\prime \prime}\left(u_{i+2}\right)=\frac{36 D_{\boldsymbol{Q}_{i-1} \boldsymbol{Q}_{i+1}}}{\left(u_{i+3}-u_{i+1}\right)\left(u_{i+3}-u_{i}\right)\left(u_{i+4}-u_{i+1}\right)},
$$

So

$$
\kappa_{i}=\frac{4\left(u_{i+3}-u_{i+1}\right)^{2} D_{\boldsymbol{Q}_{i-1} \boldsymbol{Q}_{i+1}}}{3\left(u_{i+3}-u_{i}\right)\left(u_{i+4}-u_{i+1}\right)\left\|\lambda_{i}\left(\boldsymbol{Q}_{i}-\boldsymbol{Q}_{i-1}\right)+\mu_{i}\left(\boldsymbol{Q}_{i+1}-\boldsymbol{Q}_{i}\right)\right\|^{3}},
$$

Therefore formula (4) is followed immediately from formulae (3) and (5).

Corollary 1 Let $\boldsymbol{p}_{i}(u)(i=1,2, \mathrm{~L}, n-2)$ be a uniform cubic B-spline curve segment, then

$$
K_{i}=27 \kappa_{i} / 16 .
$$

In fact, formula (6) is immediately yielded by setting $\Delta u_{i}=u_{i+1}-u_{i}$ to be a constant in formula (4) and then simplifying.

\section{Smoothing criterion based on the second discrete curvature}

In curve modeling with B-spline, the curve is usually constructed infinitely differentiable within each curve segment, but the differentiability at a node depends on repeat number of the node. Thus the fairing process of B-spline curves is the fairing process at the endpoints of B-spline curve segments corresponding to the node vector; and we need to consider the curvatures at endpoints only.

In general, people tend to take the curvatures as a directory function of curve smoothness. Intuitively, if the curvature of a curve changes slowly, then the curve is smooth [16]. Conversely, if the curvature changes rapidly, then following two case will appear: (a) The bending direction of the curve changes, then the sign of curvature changes, namely: there is an extra infection point; (b) The sign of curvature doesn't change, but the curvature values changes rapidly within a certain range, then extra bump curve segments appear. The curves are certainly not smooth if the above two cases appear.

Based on the above analysis and definition of the second discrete curvature, we believe that the curve is smooth if the number of the curve infection points and the number of curve segments are minimal under the circumstance to ensure the design requirements. Then we can determine bad points and their types by the following criteria. 
(1) To ensure the minimum number of curve infection points (preferably zero), we refer the point $\boldsymbol{Q}_{i}$ satisfying the conditions $K_{i} K_{i+1} \leq 0$ and $K_{i-1} K_{i}>0$ as a bad point of the first kind. In this case, the sign of curvature at the node $u_{i}$ changes.

(2) As an extreme curvature value corresponds to a concave (convex) curve segment, therefore we refer the point $\boldsymbol{Q}_{i}$ at which the sign of first order difference changes, namely satisfying condition $\Delta K_{i} \Delta K_{i+1}<0$ as a bad point of the second kind. In this case, the curvature in interval $\left[u_{i-1}, u_{i+1}\right]$ is non-monotonic and thus the reaches a local extreme at the node $u_{i}$.

(3) Because B-spline curves have strong convex hull property, we refer the point $\boldsymbol{Q}_{i}$ not satisfying convexity condition $K_{i}>\frac{1}{2}\left[\left(1-\tau_{i}\right) K_{i-1}+\tau_{i} K_{i+1}\right]$ as a bad point of the third kind under the circumstance to ensure constant curvature sign.

\section{Adjusting of bad points}

In this part, we denote $a=\max \left\{u_{m}, u_{3}\right\}, b=\min \left\{u_{m+4}, u_{n+1}\right\}$ and consider the local optimization algorithm which mainly minimize the energy of the curve under an acceptable change of the shape of the curve, thus the linear combination of the energy function and error function of the curve:

$$
G=(1-\delta) \sum_{i \in \Delta} \int_{0}^{\alpha}\left(\overline{\boldsymbol{p}}_{i}^{\prime \prime}(u)\right)^{2} d u+\delta \sum_{i=0}^{n} \beta_{i}\left(\boldsymbol{Q}_{i}-\overline{\boldsymbol{Q}}_{i}\right)^{2}
$$

is used as the objective function, where when $\delta \in[0,1]$.

\section{Adjusting of a control point}

Suppose $Q_{m}$ is a single bad point in the sequence of control points, then adjusting its position will lead to a shape change of the curve segment defined on interval $[a, b]$.

In other words, the adjustment of $\boldsymbol{Q}_{m}$ relate with at most the six points $\boldsymbol{Q}_{m-3}, \boldsymbol{Q}_{m-2}, \boldsymbol{Q}_{m-1}, \boldsymbol{Q}_{m+1}, \boldsymbol{Q}_{m+2}, \boldsymbol{Q}_{m+3}$. By formula (6), the point after adjusting be

$$
\overline{\boldsymbol{Q}}_{m}=\delta \boldsymbol{Q}_{m}-(1-\delta) \sum_{i=i_{0}, i \neq m}^{i_{1}} \delta_{i} \boldsymbol{Q}_{i},
$$

where $\delta \in[0,1], \delta_{i}=-\frac{\int_{a}^{b}\left(N_{i, k}^{\prime \prime}(u) N_{m, k}^{\prime \prime}(u)\right) d u}{\int_{a}^{b}\left(N_{m, k}^{\prime \prime}(u)\right)^{2} d u} ; i_{0}=\max \{0, m-3\}, i_{1}=\min \{m+3, n\}$.

\section{Adjusting of two control points}

Suppose $\boldsymbol{Q}_{m}$ and $\boldsymbol{Q}_{m+1}$ are two consecutive bad points in the sequence of the control points, adjusting their positions will lead to a shape change of the curve segment defined on interval $[a, b]$.

By formula (6), the points after adjusting satisfy the following linear equations

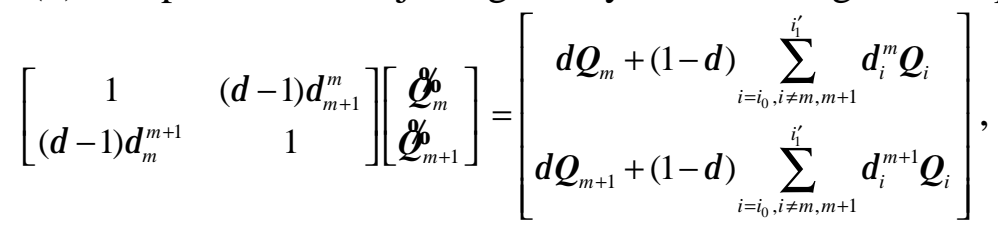

where $\delta \in[0,1], \delta_{i}^{j}=-\frac{\int_{a}^{b}\left(N_{i, k}^{\prime \prime}(u) N_{j, k}^{\prime \prime}(u)\right) d u}{\int_{a}^{b}\left(N_{j, k}^{\prime \prime}(u)\right)^{2} d u} ; i_{0}=\max \{0, m-3\}, i_{1}^{\prime}=\min \{m+4, n\}$.

Obviously, the right sides of equations (8) and (9) are the weighted averages of the original control points, and $\delta \in[0,1]$ is the modifying factor, thus the greater $\delta$, the smaller the offset of control points.

Remark 2 We find that adjusting three adjacent control points will lead the new curve to obvious bias with the shape of the original curve, so this paper only consider the algorithm of the case to adjust two adjacent control points at most. 


\section{Controlling of errors}

Theorem 3 Let $\boldsymbol{Q}_{m}$ be a bad control point of the original curve, $\overline{\boldsymbol{Q}}_{m}$ be the control point of the curve after fairing, and $\left\|\boldsymbol{Q}_{m}-\overline{\boldsymbol{Q}}_{m}\right\|<\varepsilon$, then $\|\boldsymbol{p}(u)-\overline{\boldsymbol{p}}(u)\|<\varepsilon$.

Proof By the definition and properties of B-spline curves, we have $\|\boldsymbol{p}(u)-\overline{\boldsymbol{p}}(u)\|=\left\|\sum_{i=0}^{n} \boldsymbol{Q}_{i} N_{i+3}(u)-\sum_{i=0}^{n} \overline{\boldsymbol{Q}}_{i} N_{i+3}(u)\right\|=\left\|\sum_{i=0}^{n} N_{i+3}(u)\left(\boldsymbol{Q}_{i}-\overline{\boldsymbol{Q}}_{i}\right)\right\| \leq \sum_{i=0}^{n} N_{i+3}(u)\left\|\boldsymbol{Q}_{i}-\overline{\boldsymbol{Q}_{i}}\right\|<\sum_{i=0}^{n} N_{i+3}(u) \varepsilon=\varepsilon \sum_{i=0}^{n} N_{i+3}(u)=\varepsilon$.

In order to control the error between the original curve and the smoothed curve, to any given $\varepsilon>0$, we adopt

$$
\delta=\max \left\{0,1-\varepsilon /\left\|\boldsymbol{Q}_{m}-\sum_{i \neq m, i=i_{0}}^{i=i_{i}} \delta_{i} \boldsymbol{Q}_{i}\right\|\right\},
$$

if $\boldsymbol{Q}_{m}$ is a single bad point; and adopt

$$
\delta=\max \left\{0,1-\varepsilon /\left\|\boldsymbol{Q}_{m}-\sum_{i \neq m, i=i_{0}}^{i=i^{\prime}} \delta_{i} \boldsymbol{Q}_{i}\right\|, 1-\varepsilon /\left\|\boldsymbol{Q}_{m+1}-\sum_{i \neq m+1, i=i_{0}}^{i=i_{i}^{\prime}} \delta_{i} \boldsymbol{Q}_{i}\right\|\right\},
$$

if $Q_{m}$ is the first bad point in two consecutive bad points. Then the error between the original curve and the smoothed curve can be controlled in the range of $\varepsilon$. In fact,

\section{Fairing algorithm}

$$
\left\|\overline{\boldsymbol{Q}}_{m}-\boldsymbol{Q}_{m}\right\| \leq\left\|\delta \boldsymbol{Q}_{m}+(1-\delta) \sum_{i=i_{0}, i \neq m}^{i_{i}} \delta_{i} \boldsymbol{Q}_{i}-\boldsymbol{Q}_{m}\right\|=\left\|(\delta-1)\left(\boldsymbol{Q}_{m}-\sum_{i=i_{0}, i \neq m}^{i_{i}} \delta_{i} \boldsymbol{Q}_{i}\right)\right\|=\|\delta-1\|\left\|\boldsymbol{Q}_{m}-\sum_{i=i_{0}, i \neq m}^{i_{i}} \delta_{i} \boldsymbol{Q}_{i}\right\|<\varepsilon .
$$

In many fairing algorithms, Sapidis' definition $z_{i}=\left|\frac{d \kappa}{d s}\left(u_{i}+\right)-\frac{d \kappa}{d s}\left(u_{i}-\right)\right|$ and $\xi=\sum_{i} z_{i}$ are used as partial and whole curve fairing indicator function respectively. Similarly, we define $z_{i}=D_{i}^{2}$ as a discrete local curve fairing function and $\xi=\sum_{i} D_{i}^{2}$ as whole discrete curve fairing function. Here $D_{i}$ is the second-order difference at control point $\boldsymbol{Q}_{i}$, the physical meaning of its absolute value $\left|D_{i}\right|$ is the external pressure of iron to the spline of wood. Thus, the larger (smaller) $D_{i}^{2}$, the easier (more difficult) the curve deformation at the corresponding point, and the curve is not smooth (smooth). We give the following fairing algorithm in detail:

Step 1 Enter the sequence of control points $\boldsymbol{Q}_{0}, \boldsymbol{Q}_{1}, \mathrm{~L}, \boldsymbol{Q}_{n}$ out;

Step 2 Determine bad points and their types, and calculate local fairing function value $z_{i}$ at each bad point and the overall fairing indicator function value $\xi$ of the curve;

Step 3 Order $z_{i}$ decreasingly: $z_{i_{1}} \geq z_{i_{2}} \geq \mathrm{L}$, and then modify them successively in this order;

Step 4 If $\left|i_{j}-i_{j+1}\right|>1$, only adjust point $\boldsymbol{Q}_{i_{j}}$; Otherwise, adjust two points $\boldsymbol{Q}_{i_{j}}$ and $\boldsymbol{Q}_{i_{j+1}}$;

Step5 Output a series of new control points $\mathscr{Q}_{0}^{\circ}, \mathscr{Q}_{1}^{\circ}, \mathrm{L}, \mathscr{Q}_{n}^{\circ}$, and calculate the overall fairing function value $\xi^{\prime}$ after adjust. If $\xi^{\prime}$ decreases, forward to step 6; otherwise, return step 2;

Step 6 Generate a new curve with new control points $\mathscr{Q}_{0}^{\circ}, \mathscr{Q}_{1}^{\circ} \mathrm{L}, \mathscr{Q}_{n}^{\circ}$.

\section{Experimental examples}

In this part, we choose following two common examples to verify the validity of the above algorithm.

Example 1 Take a series of control points and nodes, we obtain a rabbit curve with the constructing method of non-uniform cubic B-spline curves. Obviously it is not is smooth (Fig.1). The curve is faired by the method proposed in Section3.4, and the rabbit curve after fairing is shown in Fig.2. By comparing Fig. 3 and Fig.4, we find that not only the shape of the curve after fairing is similar to that of the original one's, but also its discrete curvature and the second discrete curvature are much smaller than those of the original one's. Hence the curve obtained by our fairing method is smoother. 


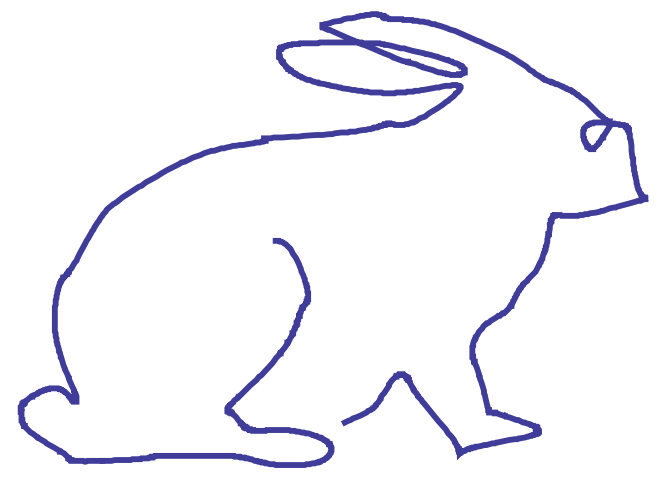

Fig. 1 Rabbit curve before fairing when $\varepsilon=1$.

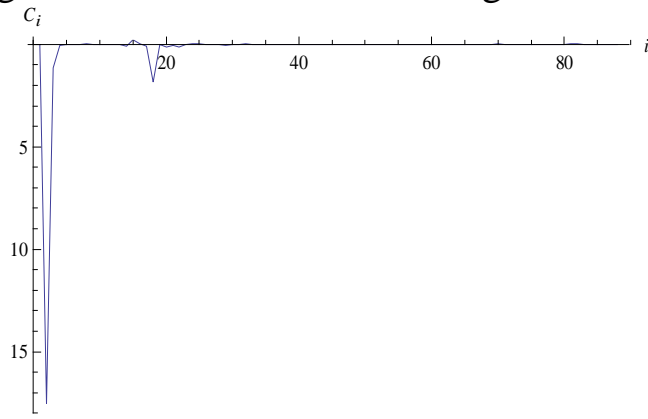

Fig. 3 The discrete curvature graph of 88 control points after fairing

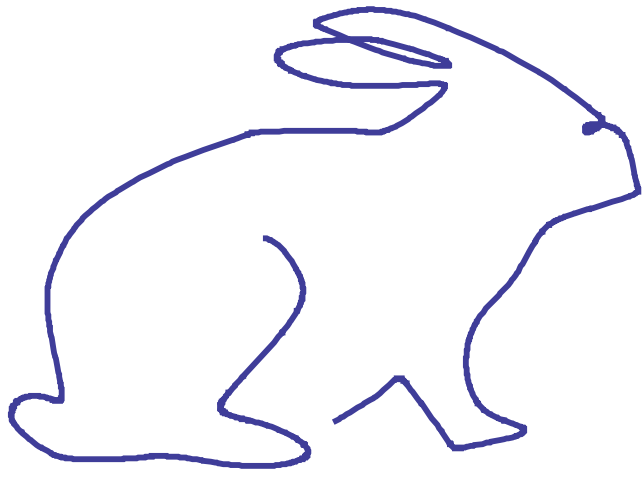

Fig.2 Rabbit carve after fairing when $\varepsilon=1$.

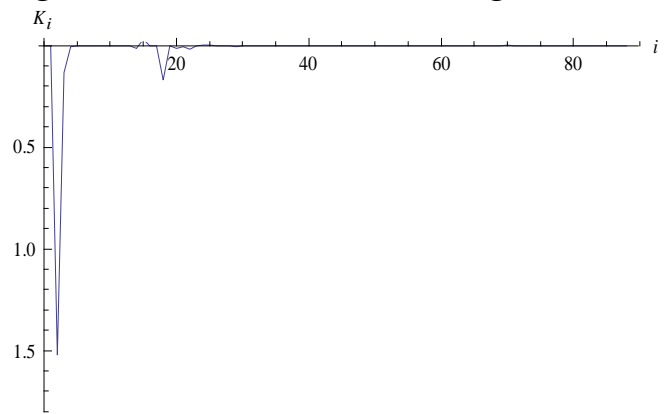

Fig.4 The second discrete curvature graph of 88 control points after fairing

Example 2 Take a series of control points and nodes, we obtain an $\mathrm{S}$ curve with the constructing method of non-uniform cubic B-spline curves. Fig. 5 to Fig. 10 are the curvature comparison graphs of original curves and the curves which are faired by the method of section 3.4, where $\varepsilon$ takes $0.2,1,5$ respectively. We find that the greater $\varepsilon$, the more gentle the curvature change of the curve, thus the better the curvature monotonicity and smoother the curve, but the greater shape change of the curve.

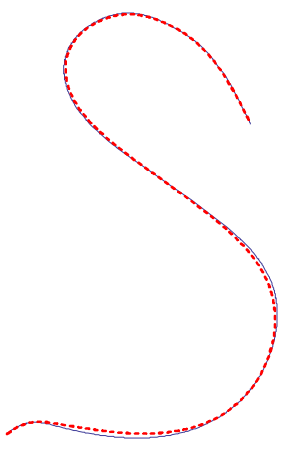

Fig.5 Comparing graph of the curve of S type before and after fairing when $\varepsilon=0.2$.

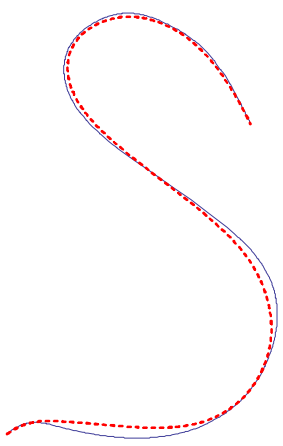

Fig.7 Comparing graph of the curve of $\mathrm{S}$ type before and after fairing when $\boldsymbol{w}_{\varepsilon=1}$.

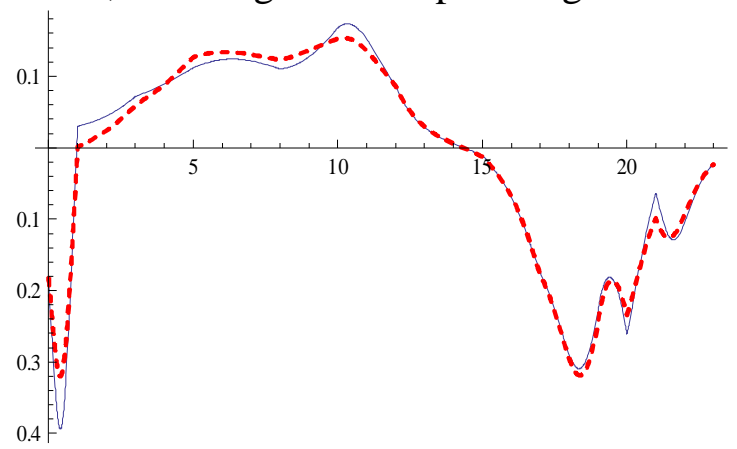

Fig.6 Curvature Comparing graph of the curve of $S$ type before and after fairing when $\varepsilon$ takes $\varepsilon=0.2$.

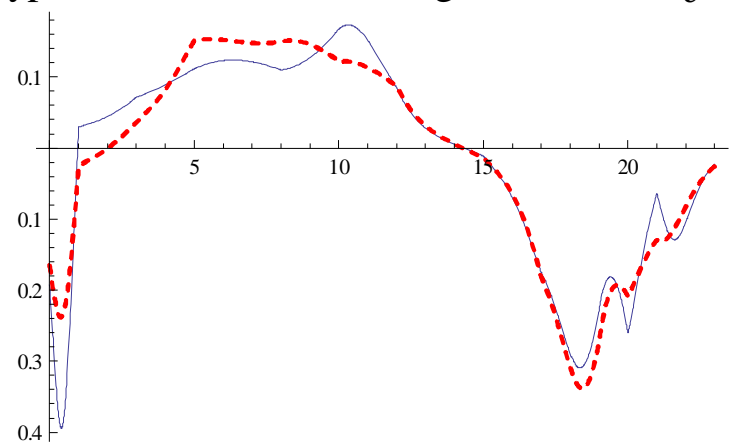

Fig.8 Curvature Comparing graph of the curve of $S$ type before and after fairing when w $_{\varepsilon}=1$. 


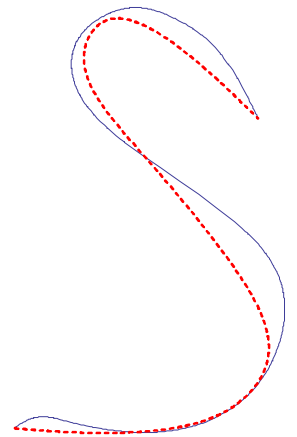

Fig.9 Comparing graph of the curve of $S$ type before and after fairing when $\varepsilon=5$.

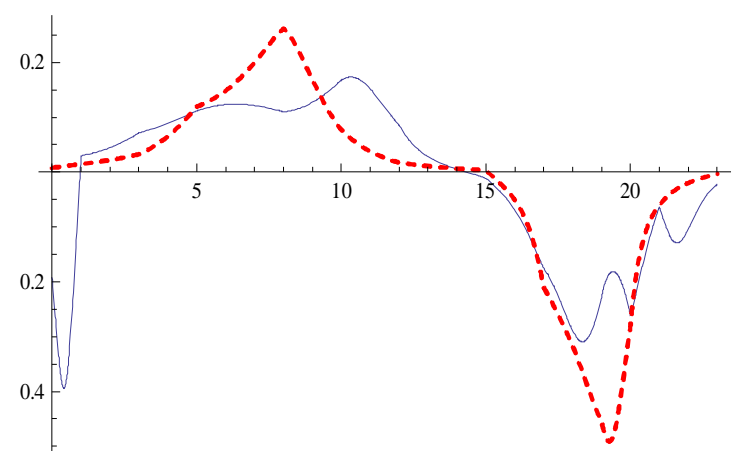

Fig.10 Curvature Comparing graph of the curve of $S$ type before and after fairing when $\mathrm{e}_{\varepsilon}=5$.

\section{Conclusions}

There are many faring methods for B-spline curves. In this paper, the concept of the second discrete curvature is put forward, the properties of the second discrete curvature and its differences are investigated. The fairing algorithm based on the second discrete curvature for cubic B-spline curves is given. This faring algorithm has unique advantages compared with traditional ones. Firstly, this method is simple and direct, just by modifying bad points in the control points of non-uniform cubic B-spline curves, the fairing purpose can be achieved; Secondly, the fairing algorithm has stronger geometric intuition. As the second discrete curvature is an expression of the triangle's area and its edge lengths, it is a geometric invariant, so its geometric intuition is stronger. In addition, since B-spline surfaces are tensor product of B-spline curves, the results of B-spline curves can be extended to B-spline surfaces.

\section{References}

[1] J. F. Poliakoff, Y K. Wong. P. D. Thomas. An automated curve fairing algorithm for cubic B-spline curves [J]. Journal of Computational and Applied Mathematics, 1999, 102:73-85

[2] Yulin Wang, Bingyan Zhao, Luzhou Zhang, Jiachuan Xu, Kanchang Wang, Shuchun Wang . Designing fair curves using monotone curvature pieces [J]. Computer Aided Geometric Design, 2004, 21: $512-527$.

[3] Weishi L, Shuhong X, Jianmin J, Zhao G. Target curvature driven fairing algorithm for planar cubic B-spline curves [J]. Computer Aided Design, 2004, 21: 499-513.

[4] Glen Mulineux, Sebastian T.Robinson, Fairing points sets using curvature [J]. Computer Aided Geometric Design, 2007, 39: 27 - 34

[5] Ján Glasa, Least-squares smoothing of 3D digital curves [J]. Real-Time Imaging, 2005, 11:465-473.

[6] Xunnan Yang, Guozhao Wang. Planar point set fairing and fitting by arc splines [J]. Computer Aided Geometric Design, 2001, 33:35-43.

[7] Robet J. Renka. Shape-preserving interpolation by fair discrete $\mathrm{G}^{3}$ space curves [J]. Computer Aided Geometric Design, 2005, 22: 793 - 809.

[8] Eck, M., Hadenfeld, J. Local energy fairing of B-spline curves [J]. Computing Supplementum, 1995, 10:129-147.

[9] Xin Yunlong. On Fitting and Faring of Curves [J]. Journal of Fudan University, Science Edition, 1975, 2:103-110.

[10] Caiming Zhang, Pifu Zhang, Fuhua Cheng. Fairing spline curves and surfaces by minimizing energy [J]. Computer Aided Design, 2001, 33 (13): 913- 923. 
[11] Liu GH, Wong YS, Zhang YF, Loh HT. Adaptive fairing of digitized point data with discrete curvature. Computer Aided Design, 2002, 34:309-320.

[12] Yu Desheng, Cheng Cheng, On Local Fairing Algorithm of Cubic Uniform B-spline with Discrete Curvature. Jounal of Zhejiang University (Science Edition), 2011,38(5):511-517.

[13] Carmel E, Cohen-Or D. Warp-guided object-space morphing [J]. The Visual Computer, 1997, 13: 465-478.

[14] Yu Desheng. On Some Theorems of Directed Areas of Polygons [J], Journal of Gannan Teacher's College, 1999(3):11-14.

[15] Wang Renhong, Li Chongjun, Zhu Chungang, et al. A Course in Computational Geometry [M]. Beijing: Science Press, 2008.

[16] Dong Guangchang, Zhang Dan, Liu Zhicheng, MA Lizhuang. Curve fairing [J], Progress in Natural Science, 1997, 7(5):525-538 